\title{
Correction to: 'National Level New Areas' and Urban Districts: Centralization of Territorial Power Relations in China
}

\section{Miguel Hidalgo Martinez ${ }^{1}[$}

Published online: 25 April 2018

(C) Fudan University and Springer Nature Singapore Pte Ltd. 2018

Correction to: Chin. Polit. Sci. Rev. https://doi.org/10.1007/s41111-018-0102-6

The original article has been corrected.

In the presentation of Table 2 two years were missing: TFNA (20H) JPNA (20H). The correct version is given here: TFNA (2014) JPNA (2014).

The original article can be found online at https://doi.org/10.1007/s41111-018-0102-6.

Miguel Hidalgo Martinez

Miguel.Martinez@xjtlu.edu.cn

1 Xi'an Jiaotong - Liverpool University, 111 Ren'ai Road, South Campus, Suzhou Dushu Lake Science and Education Innovation District, Suzhou Industrial Park, Suzhou, Jiangsu 215123, People's Republic of China 